\title{
ISOPARAMETRIC FUNCTIONS AND SUBMANIFOLDS
}

\author{
by S. M. B. KASHANI
}

(Received 7 May, 1991)

Introduction. The theory of isoparametric functions and a family of isoparametric hypersurfaces began essentially with E. Cartan in 1930's. He defined a real valued function $V$ defined on a Riemannian space form to be isoparametric if $\|\operatorname{grad} v\|^{2}=T \circ V$ and $\Delta V=S \circ V$ for some real valued functions $S, T$. Then a family of hypersurfaces $M_{t}$ is called isoparametric if $M_{t}=V^{-1}(t)$ where $t$ is a regular value of $V$.

Equivalently an isoparametric (family of) hypersurface(s) can be characterized as a (family of parallel) hypersurface(s), (each of) which has constant principal curvature. The isoparametric submanifolds in $\mathbb{B}^{N}$ and $H^{N}$ are almost trivial, but in $S^{N}$ the study of such submanifolds is quite interesting and many mathematicians have contributed to the subject. The generalization of this subject to higher dimensions was done by Carter-West [1] and Terng [5].

Nomizu [4] and Magid and Hahn [2] generalized the notion of isoparametric hypersurface and function to semi-Riemannian spaces. In this paper we generalize their work and study the notion of isoparametric submanifolds and maps of codimension at least 2 in semi-Riemannian spaces.

We observe that there are some similarities and many crucial differences between the theory in $\mathbb{R}^{N}$ and in $\mathbb{R}_{p}^{N}$. In fact the tangent bundle $T M$ of each isoparametric submanifold $M$ in $\mathbb{R}^{N}$ has a decomposition as $T M=\bigoplus_{i=1}^{k} E_{i}$ where each $E_{i}$ is an integrable distribution. The principal curvatures of $M$ are all real with fixed algbraic multiplicities on $M$. Each $E_{i}$ is generated by eigenvectors of the principal curvatures of $M$. Using these facts one gets a finite reflection group (the Coxeter group of $M$ ) acting on the normal bundle of $M$. This is the key to the study of isoparametric submanifolds in $\mathbb{R}^{N}$. By using this group one can prove many important facts about such submanifolds. For example, the reducibility of each isoparametric into the product of irreducible ones, the fact that each isoparametric submanifold $M$ in $\mathbb{R}^{N}$ is algebraic, the classification of isoparametric submanifolds.

All these results need serious investigation for an isoparametric submanifold $M$ in $\mathbb{R}_{p}^{N}$. Since in sharp contrast to the Riemannian case the principal curvatures of $M$ are not real (in general). The algebraic and geometric multiplicities of a real (if it exists) principal curvature can be different. The geometric multiplicity of a real principal curvature can vary by varying the point $x$ in $M$, etc. A major consequence of these facts is that isoparametric submanifolds in $\mathbb{R}_{p}^{N}$ are not in general algebraic. This is one of the crucial differences between the theory in $\mathbb{R}^{N}$ and in $\mathbb{R}_{p}^{N}$. An example of a nonalgebraic isoparametric submanifold in $\mathbb{R}_{p}^{N}$ is given at the end of the paper. Another consequence of the above facts is that in general we do not have a decomposition of $T M$ into integrable distributions, but we just have some partial results in this direction. So the lack of such a decomposition (in general) which results from the lack of any group (similar to the Coxeter group) associated to $M$ makes the study of isoparametric submanifolds in $\mathbb{R}_{p}^{N}$ quite different and more difficult than the study of such submanifolds in $\mathbb{R}^{N}$, and as mentioned above not all the facts which are true for isoparametric submanifolds in $\mathbb{B}^{N}$ can be generalized to similar facts for such submanifolds in $\mathbb{R}_{p}^{N}$. Throughout the paper we have pointed out the differences quite explicitly.

Glasgow Math. J. 35 (1993) 145-152. 


\section{Main Results.}

Definition 1. A smooth function $f=\left(f_{n+1}, \ldots, f_{n+m}\right): \mathbb{R}_{p}^{n+m} \rightarrow \mathbb{R}^{m}$ is called isoparametric if

(i) $\left\langle\operatorname{gr} f_{\alpha}, \operatorname{gr} f_{\beta}\right\rangle$ and $\Delta f_{\alpha}=\operatorname{div}\left(\operatorname{gr} f_{\alpha}\right)$ are (continuous) functions of $f$ for each $\alpha, \beta$; $n+1 \leq \alpha, \beta \leq n+m$,

(ii) $\left[\operatorname{gr} f_{\alpha}, \operatorname{gr} f_{\beta}\right.$ ] is a linear combination of $\operatorname{gr} f_{n+1}, \ldots, \operatorname{gr} f_{n+m}$ with coefficients being continuous functions of $f$ for each $\alpha, \beta ; n+1 \leq \alpha, \beta \leq n+m$.

Definition 2. A connected semi-Riemannian submanifold $M^{n}$ of $\mathbb{R}_{p}^{n+m}$ is called isoparametric if

(i) the normal bundle $N M$ is flat with trivial holonomy group,

(ii) the characteristic polynomial of the shape operator of $M$ along any parallel normal vector field is the same at all points of $M$.

THEOREM 3. Let $f: \mathbb{R}_{p}^{n+m} \rightarrow \mathbb{R}^{m}$ be an isoparametric function, $c$ a regular value of $f$ such that $\phi \neq f^{-1}(c)$ and $\left.\langle\rangle\right|_{T,\left(f^{-1}(c)\right)}$ is nondegenerate. Let $M$ be a connected component of $f^{-1}(c)$; then $M$ is an isoparametric submanifold.

Proof. The proof is very much like the Riemannian case (see [1] or [5]) and we give just a sketch of it. We prove (i) by finding a global parallel normal orthonormal frame field $\left\{e_{\alpha}\right\}$ on $M$. In order to prove (ii) we show first that the mean curvature vector field of $M$, i.e., $H=\frac{1}{n} \sum_{i=1}^{n} \varepsilon_{i} I I\left(e_{i}, e_{i}\right)=\sum_{\alpha} H_{\alpha} e_{\alpha}\left(\left\{e_{i}\right\}\right.$ is any (local) orthonormal tangent frame field on $M$ ) is parallel on $M$ or, equivalently, each mean curvature $H_{\alpha}$ is constant on $M$. Then we get the benefit of parallel surfaces $M_{t}$ of $M$. Explicitly by choosing a parallel normal vector field $e_{\alpha}$ on $M$ and pushing out each point of $M$ in the $e_{\alpha}$ direction we get the map $\varphi_{t}: M \rightarrow \mathbb{R}_{p}^{n+m}$ defined by $\varphi_{t}(x)=x+t e_{\alpha}(x)$. If $t$ is small enough then (locally) $M_{t}=\varphi_{t}(M)$ is an $n$-dimensional immersed submanifold of $\mathbb{R}_{p}^{n+m}$ which has a global parallel normal orthonormal frame field and its mean curvature with respect to any parallel normal vector field is constant on $\boldsymbol{M}_{\boldsymbol{t}}$. Then by using Nomizu's method in [4] in our context which says that "a hypersurface $N_{0}$ has constant principal curvature if each of its parallel surfaces $N_{t}$ (for small $t$ ) has constant mean curvature" we get (ii).

Remark 4. We see that each $M_{t}$, as defined above, is an isoparametric submanifold. The one parameter family $\left\{M_{t}\right\}$ is called the isoparametric system associated with $M$ in the $e_{\alpha}$ direction; note that $M_{0}=M$.

The next two propositions deal with the curvature foliation on $M$.

Proposition 5. Let $M^{n}$ be an isoparametric submanifold of $\mathbb{R}_{p}^{n+m}$, let $\xi$ be a parallel unit $(\langle\xi, \xi\rangle= \pm 1)$ normal vector field on $M$ and $S$ the shape operator of $M$ along $\xi$. If $K$ is a real eigenvalue of $S, T_{k}=\operatorname{Ker}\left(S-K I_{n}\right)$ and if the dimension of $T_{k}$ (=geometric multiplicity of $K$ ) is a fixed number $l$, then

(i) $T_{k}$ is integrable,

(ii) if $T_{k}$ is nondegenerate, the integral manifold $M_{k}$ of $T_{k}$ is totally geodesic in $M$,

(iii) if the algebraic multiplicity of $K$ is equal to its geometric multiplicity, then $T_{k}$ is nondegenerate. 
Proof. (i) if $X, Y \in T_{k}$, by using the Codazzi equation $\left(\left(\nabla_{v} I I\right)(U, W)=\left(\nabla_{u} I I\right)\right.$ $(V, W) \forall U, V, W \in X(M))$ we prove that $\nabla_{X} Y \in T_{k}$. Thus $[X, Y] \in T$, i.e., $T_{k}$ is integrable.

(ii) In this case $M$ is a semi-Riemannian submanifold of $M$ and by part (i) we see that the second fundamental form of $M_{k}$ in $M$ is identically zero, hence $M_{k}$ is totally geodesic in $M$.

(iii) Let the characteristic polynomial of $S$ be $(t-K)^{r_{1}}\left(p_{2}(t)\right)^{r_{2}} \cdots\left(p_{s}(t)\right)^{r_{s}}$, where each $p_{i}(t)$ is an irreducible polynomial in $R[t]$. We know that

$$
T M=\operatorname{Ker}(S-K I)^{r_{1}} \oplus \operatorname{Ker}\left(p_{2}(S)\right)^{r_{2}} \oplus \ldots \oplus \operatorname{Ker}\left(p_{s}(S)\right)^{r_{s}} .
$$

These kernels are mutually orthogonal, hence each one is nondegenerate. If the algebraic multiplicity of $K$ is equal to its geometric multiplicity, the $\operatorname{Ker}(S-K I)=\operatorname{Ker}(S+K I)^{r_{1}}$. Thus $\operatorname{Ker}(S-K I)$ is nondegenerate.

Proposition 6. Let $M^{n} \subset \mathbb{R}_{p}^{n+m}$ be a (geodesically) complete isoparametric submanifold, let $\left\{e_{\alpha}\right\}$ be a parallel normal orthonormal frame field on $M$ and $S_{\alpha}$ the corresponding shape operator of $M$, let the characteristic polynomial of each $S_{\alpha}$ be $p_{\alpha}(t)=\left(t-K_{\alpha}\right)^{r_{\alpha, 1}}\left(p_{\alpha, 2}(t)\right)^{r_{\alpha .2}} \ldots\left(p_{\alpha, s_{\alpha}}(t)\right)^{r_{\alpha} s_{\alpha}}$ and put $T_{K_{\alpha}}=\left\{X \in X(M): S_{\alpha} X=K_{\alpha} X\right\}$. Suppose that $V=\bigcap_{\alpha} T_{K_{\alpha}}$ is a nondegenerate 2 -dimensional subbundle of $T M(l \geq 1)$. Put $v=\sum_{\alpha} \varepsilon_{\alpha} K_{\alpha} e_{\alpha} \in K^{\perp}(M) ;$ then

(i) $V$ is integrable and its integral manifold $L$ is totally geodesic in $M$,

(ii) if $v=0$, the integral manifold $L$ of $V$ through $x \in M$ is the plane $x+\mathbb{R}_{s}^{\prime}$ in $\mathbb{R}_{p}^{n+m}$, where $s$ is the index of $\left.\langle\rangle\right|_{v$,$} ,$

(iii) if $\langle v, v\rangle \neq 0, L$ is (a component of) an l-dimensional sphere or pseudosphere if $\langle v, v\rangle>0$ or pseudohyperbolic space if $\langle v, v\rangle<0$ with radius $\frac{1}{|\langle v, v\rangle|}$ and centre $c=y+\frac{v(y)}{\langle v, v\rangle} \forall y \in L$

Proof. (i) By Proposition 5(i), if $X, Y \in V$, then $\nabla_{X} Y \in V$ so $V$ is integrable and its integral manifold $L$ is totally geodesic in $M$.

(ii) Since $v=0, K_{\alpha}=0$ for all $\alpha, n+1 \leq \alpha \leq n+m$, and the second fundamental form of $L$ in $\mathbb{R}_{p}^{n+m}$ is $I_{L}(X, Y)=I_{L, 1}(X, Y)+I_{L, 2}(X, Y)$ where $I_{L, 1}(X, Y) \in T M / V$ and $I_{L, 2}(X, Y) \in X^{\perp}(M)$ for each $X, Y \in V$. In (i) we proved that $I_{L, 1}(X, Y)=0$, we also have $0=\left\langle S_{\alpha} X, Y\right\rangle=\left\langle I_{L, 2}(X, Y), e_{\alpha}\right\rangle$ for each $\alpha$, and $X, Y \in V$, so $I_{L, 2}(X, Y)=0$, thus $I_{L}(X, Y)=0$. Hence $L$ is totally geodesic in $\mathbb{R}_{p}^{n+m}$, since $M$ is complete, $L$ is isometric to a plane $x+\mathbb{R}_{s}^{\prime} \subset \mathbb{R}_{p}^{n+m}$.

(iii) We see that the map $\varphi(y)=y+\frac{v(y)}{\langle v, v\rangle}$ maps $L$ to a constant vector $c \in \mathbb{R}_{p}^{n+m}$ and that $I_{L}(X, Y)=\langle X, Y\rangle v, \forall X, Y \in V$, i.e. $L$ is totally umbilic in $\mathbb{R}_{p}^{n+m}$. The claim is obtained from these two facts.

Definition 7. Let $M^{n} \subset \mathbb{R}_{p}^{n+m}$ be a semi-Riemannian submanifold and $\left\{v_{\alpha}\right\}$ be a normal frame field on $M$, let $\varphi: M \times \mathbb{R}^{m} \rightarrow \mathbb{R}_{p}^{n+m}$ be defined by $\varphi(x, z)=x+\sum_{\alpha} z_{\alpha} v_{\alpha}(x)$ 
$\forall x \in M, \forall z=\left(z_{n+1}, \ldots, z_{n+m}\right) \in \mathbb{R}^{m}$. A point $e=x+\sum_{\alpha} z_{\alpha} v_{\alpha}(x)$ is called a focal point of $M$ if $e$ is a critical value of $\varphi$ (i.e., the Jacobian of $\varphi$ at some point $(x, z) \in \varphi^{-1}(e)$ is singular). The set of all focal points of $M$ is called the focal set of $M$. If $z \in \mathbb{R}^{m}$ is such that for each point $x \in M, \varphi(x, z)$ is a critical value of $\varphi$, then $\varphi(M \times\{z\})$ is called a focal manifold of $M$ associated to $z,\left\{v_{\alpha}\right\}$.

Proposition 8. Let $M^{n}$ be an isoparametric submanifold of $\mathbb{R}_{p}^{n+m}$, $\xi$ be a (local) parallel normal vector field on $M,\langle\xi, \xi\rangle \neq 0, S$ the shape operator of $M$ along $\xi$ which has $K$ as a non-zero real eigenvalue.

(i) If $K$ has multiplicity $l$, define $\varphi: M^{n} \rightarrow \mathbb{R}_{p}^{n+m}$ by $\varphi(x)=x+\frac{1}{K} \xi(x)$ then $\varphi$ is a submersion of $M$ (at least locally) onto a nondegenerate submanifold of $\mathbb{R}_{p}^{n+m}$ of codimension $l+m$ that will be denoted by $V_{K}$ and is the focal manifold of $M$ associated with $\xi, K$.

If $\left(s_{K}, l-s_{K}\right)$ is the signature of $T_{K}$, the eigendistribution of $S$ associated with $K$, then $V_{K}$ has signature $\left(s-s_{K}, n+s_{K}-l-s\right)$. The integral submanifold $M_{K}$ of $T_{K}$ through $x \in M$ is mapped by $\varphi$ onto the single point $\varphi(x)$.

If the shape operator of $M$ at $x$ is given by $S=\left[\begin{array}{c|c}K I_{I} & 0 \\ \hline 0 & A\end{array}\right]$ relative to the orthogonal decomposition $T_{z} M=T_{K}(x) \oplus\left(T_{K}(x)\right)^{\perp}$, then the shape operator of $V_{K}$ associated with $\xi^{K}=\xi\left(\xi^{K}\right.$ is the parallel transport of $\xi$ along the curve $\left.t \mapsto x+t \xi(x)\right)$ is $S_{K}=$ $A\left(I_{n-1}-\frac{1}{k} A\right)^{-1}$.

(ii) If the geometric multiplicity of $K$ is constant but different from its algebraic multiplicity, then $\varphi$ is a submersion onto a submanifold with degenerate metric.

(iii) If $\varphi_{t}: M \rightarrow \mathbb{R}_{p}^{n+m}$ is defined by $\varphi_{t}(x)=x+\frac{1}{t} \xi(x)$ and $\varphi_{t}(M)=V_{t}$ is a submanifold with nondegenerate metric of signature $\left(s-s_{t}, n+s_{t}-l-s\right), l>0$, then $t$ is a principal curvature of $M$ of multiplicity $l$ and $V_{t}$ is the focal manifold of $M$ associated with $\xi$, $t$. The eigendistribution $T_{t}$ has signature $\left(s_{t}, l-s_{t}\right)$, the shape operator of $M$ along $\xi$ at $x$ is given by

$$
S_{\xi}=\left[\begin{array}{l|l}
t I_{l} & 0 \\
\hline 0 & A
\end{array}\right]
$$

relative to the orthogonal decomposition $T_{z} M=T_{K}(x) \oplus\left(T_{k}(x)\right)^{\perp}$, where $A$ is $t\left(t I_{n-1}+S_{t}\right)^{-1} S_{t}$ and $S_{t}$ is the shape operator of $V_{t}$ along $\xi^{t}$.

Proof. The proofs of (i) and (iii) are almost the same as in the Riemannian case. For (ii) we suppose that the claim is false, i.e., $T V_{K}$ is nondegenerate, then its normal bundle

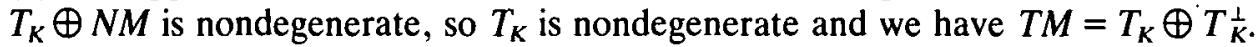

By using the fact that algebraic and geometric multiplicities of $K$ are different and representing the shape operator of $M$ with respect to a basis adapted to the decomposition $T M=T_{K} \oplus T_{\mathcal{K}}^{\perp}$ we get that $T_{K}$ is degenerate, which is a contradiction, hence $T V_{K}$ must be degenerate. 
Here we look at another problem about isoparametric submanifolds. The problem concerns the product of two isoparametric submanifolds. The proof of the following proposition is exactly the same as in the Riemannian case.

Proposition 9. If $M_{i}^{n_{i}}$ is an isoparametric submanifold in $\mathbb{R}_{p_{i}}^{n_{i}+l_{i}}, i=1,2$, then $M_{1} \times M_{2}$ is isoparametric in $\mathbb{R}_{p_{1}+p_{2}+n_{1}+l_{2}}^{n_{1}+l_{2}}$.

REMARK 10. The converse of the above proposition is far from being obvious, that is the question of when an isoparametric submanifold $M^{n} \subset \mathbb{R}_{p}^{n+m}$ decomposes into two lower dimensional isoparametric submanifolds is open and needs a serious investigation. The solution to this problem is well known in the Riemannian case, in fact if $M^{n} \subset \mathbb{R}^{n+m}$ (or $S^{n+m}$ or $H_{0}^{n+m}$ ) is an isoparametric submanifold, then it decomposes into "irreducible" isoparametric submanifolds if and only if the Coxeter group of $\boldsymbol{M}$ decomposes into irreducible subgroups. In contrast, in $\mathbb{R}_{p}^{n+m}$ we have no group associated to the isoparametric submanifold $M^{n} \subset \mathbb{R}_{p}^{n+m}$.

REMARK 11. We defined an isoparametric submanifold to be in $\mathbb{R}_{p}^{n+m}$ and an isoparametric map to have $\mathbb{R}_{p}^{n+m}$ as its domain. We can define an isoparametric submanifold in a component of $S_{p}^{n+m}$ or $H_{p}^{n+m}$, and an isoparametric map with domain $S_{p}^{n+m}$ or $H_{p}^{n+m}$ exactly as we did for $\mathbb{R}_{p}^{n+m}$. Then almost all the material of the paper goes through with only slight changes.

Remark 12. It is a well-known fact that, if $M^{n} \subset \mathbb{R}^{n+m}$ is an isoparametric submanifold, there exists a polynomial map $f: \mathbb{R}^{n+m} \rightarrow \mathbb{R}^{m}$ such that $f$ is isoparametric and $M$ is a regular level of $f$. The situation in $\mathbb{R}_{p}^{n+m}$ is far from being obvious and the following example shows that, in contrast to the Riemannian case, in general we fail to have such an $f$. So the questions in $\mathbb{R}_{p}^{n+m}$ are as follows.

If $M^{n} \subset \mathbb{R}_{p}^{n+m}$ is an isoparametric submanifold, is there any isoparametric map $f: \mathbb{R}_{p}^{n+m} \rightarrow \mathbb{R}^{m}$ such that $M$ is a component of a nondegenerate regular level $f^{-1}(c)$ ? A weaker question is under what conditions there is an isoparametric function $f: \mathbb{R}_{p}^{n+m} \rightarrow$ $\mathbb{R}^{m}$ such that $M$ is a component of $f^{-1}(c)$ ?

EXAMPLE 13. In this example we illustrate an isoparametric submanifold $M^{2} \subset \mathbb{R}_{1}^{4}$ which is not algebraic.

Let $A=\frac{1}{\sqrt{2}}(1,-1,0,0), B=\frac{1}{\sqrt{2}}(1,1,0,0), C_{1}=(0,0,1,0), C_{2}=(0,0,0,1)$ and define $M^{2} \subset \mathbb{R}_{1}^{4}$ by $M=\left\{\psi(s, t)=t A+C_{1} \sin t+t C_{2}+s B: s, t \in \mathbb{R}\right\}=\left\{x=\left(\frac{1}{\sqrt{2}}(t+s)\right.\right.$, $\left.\left.\frac{1}{\sqrt{2}}(-t+s), \sin t, t\right): t, s \in \mathbb{R}\right\}$. Then $\frac{\partial \psi}{\partial s}=B, \frac{\partial \psi}{\partial t}=A+C_{2}+C_{1} \cos t$ is a frame field for $T M$ and $\left\{B \cos t+C_{1}, C_{2}+B\right\}$ is a frame field for $N M$. Let $S_{1}, S_{2}$ be shape operators of $M$ along $\left(B \cos t+C_{1}\right)$ and $\left(C_{2}+B\right)$ respectively. Obviously $S_{2} \equiv 0$, by a routine calculation we see that $S_{1} B=0$ and $S_{1}\left(A+C_{2}+C_{1} \cos t\right)=4 B \sin T$. So with respect to the chosen tangent frame field

$$
S_{1}=\left[\begin{array}{cc}
0 & 4 \sin t \\
0 & 0
\end{array}\right]
$$


The calculation also shows that $M$ has a global parallel normal orthonormal frame field. As can be seen, the principal curvature (and its algebraic multiplicity) of $M$ is constant on $M$ in any parallel normal direction. Thus $M$ is isoparametric. The example is interesting from different points of view. The geometric multiplicity of the eigenvalue of $S_{1}$ is not constant on $M$. Thus the minimal polynomial of $S_{1}$ is not the same at all points of $M . M$ is not algebraic since if there exists a polynomial map $f: \mathbb{R}_{1}^{4} \rightarrow \mathbb{R}^{2}$ such that $M$ is a component of $f^{-1}(c)$, then $\mathrm{gr} f_{1}, \operatorname{gr} f_{2}$ must be polynomial functions in $x=\left(x_{1}, \ldots, x_{4}\right) \epsilon$ $M$. Thus $B \cos t+C_{1}$ must be a polynomial function of $x$, which certainly it is not, in fact

$$
B \cos t+C_{1}=\left(\sqrt{\frac{1-x_{3}^{2}}{2}}, \sqrt{\frac{1-x_{3}^{2}}{2}}, 1,0\right)
$$

The following is an interesting example of an isoparametric map $f: \mathbb{R}_{3 p}^{3 n} \rightarrow \mathbb{R}^{3}$. It is the only nontrivial one (except the quadratics) which we have found.

Example 14. Let $\bar{M}$ be the space of $3 \times n$ matrices over $\mathbb{R}$, define the scalar product on $\bar{M}$ by $\langle x, y\rangle=\operatorname{tr} x J y^{t}$, where $S^{t}$ means the transpose of the matrix $S$ and

$$
J=\left[\begin{array}{c|c}
-I_{p} & 0 \\
\hline 0 & I_{n-p}
\end{array}\right] .
$$

Define $f_{r} ; \bar{M} \rightarrow \mathbb{R}$ by $f_{r}(x)=\operatorname{tr}\left(x J x^{t}\right)^{r}$ for $r \in Z_{+}$. Let us check that the map $f: \bar{M} \rightarrow \mathbb{B}^{3}$ defined by $f(x)=\left(f_{1}(x), f_{2}(x), f_{3}(x)\right)$ is an isoparametric map.

Proof. Easily we get that $\operatorname{gr} f_{1}(x)=2 x$, gr $f_{2}(x)=4 x J x^{t} x$ and $\operatorname{gr} f_{3}=6\left(x J x^{t}\right)^{2} x$. Thus $\left\langle\operatorname{gr} f_{1}, \operatorname{gr} f_{1}\right\rangle=4 f_{1}, \quad\left\langle\operatorname{gr} f_{1}, \operatorname{gr} f_{2}\right\rangle=8 f_{2}, \quad\left\langle\operatorname{gr} f_{1}, \operatorname{gr} f_{3}\right\rangle=16 f_{3}, \quad\left\langle\operatorname{gr} f_{2}, \operatorname{gr} f_{2}\right\rangle=16 f_{3}$, $\left\langle\operatorname{gr} f_{2}, \operatorname{gr} f_{3}\right\rangle=24 f_{4},\left\langle\operatorname{gr} f_{2}, \operatorname{gr} f_{3}\right\rangle=36 f_{5}$.

We must prove that $f_{4}$ and $f_{5}$ are functions of $f_{1}, f_{2}, f_{3}$. Since $\left(x J x^{t}\right)^{r}$ is a real symmetric matrix for each $r \in Z_{+}$, it can be brought into diagonal form. So $\left(x J x^{t}\right)^{r}$ is similar to

$$
\left[\begin{array}{ccc}
(\lambda(x))^{r} & & 0 \\
0 & (\mu(x))^{r} & 0 \\
0 & & (v(x))^{r}
\end{array}\right]
$$

for some $\lambda(x), \mu(x), v(x) \in \mathbb{R}$. Thus $f_{r}=(\lambda(x))^{r}+(\mu(x))^{r}+(v(x))^{r}$. By using Newton's formulae for elementary symmetric polynomials in three variables $\lambda, \mu, v$ we get that $f_{4}=\frac{1}{6}\left(f_{1}^{4}+3 f_{2}^{2}-6 f_{1}^{2} f_{2}+8 f_{1} f_{2}\right)$. Hence $\left\langle\operatorname{gr} f_{2}\right.$, gr $\left.f_{3}\right\rangle=24 f_{4}$ can be expressed in terms of $f_{1}, f_{2}, f_{3}$. Similarly we obtain that $f_{5}=\frac{1}{6}\left(f_{1}^{5}-5 f_{1}^{3} f_{2}+5 f_{1}^{2} f_{3}+5 f_{2} f_{3}\right)$, so $\left\langle\operatorname{gr} f_{3}, \operatorname{gr} f_{3}\right\rangle=36 f_{5}$ can be expressed in terms of $f_{1}, f_{2}, f_{3}$.

Now we are going to look at $\left[\mathrm{gr} f_{\alpha}, \operatorname{gr} f_{\beta}\right]$. Let $D$ be the Levi Civita connection in $\bar{M} \cong \mathbb{R}_{3 p}^{3 n}$ then we have $\left[\operatorname{gr} f_{\alpha}, \operatorname{gr} f_{\beta}\right]=D_{\operatorname{gr} f_{\alpha}} \operatorname{gr} f_{\beta}-D_{\operatorname{grf} f_{\beta}} \operatorname{gr} f_{\alpha}$, so $\left[\operatorname{gr} f_{1}, \operatorname{gr} f_{2}\right]=4 \operatorname{gr} f_{2}$, $\left[\operatorname{gr} f_{1}, \operatorname{gr} f_{3}\right]=8 \operatorname{gr} f_{3},\left[\operatorname{gr} f_{2}, \operatorname{gr} f_{3}\right]=48\left(x J x^{t}\right)^{3} x=6 \operatorname{gr} f_{4}$. By using the relation $f_{4}=\frac{1}{6}\left(f_{1}^{4}+\right.$ $\left.3 f_{2}^{2}-6 f_{1}^{2} f_{2}+8 f_{1} f_{3}\right)$ we get that $\left[\mathrm{gr} f_{2}, \operatorname{gr} f_{3}\right]=\left(4 f_{1}^{3} \operatorname{gr} f_{1}+6 f_{2} \operatorname{gr} f_{2}-12 f_{1} f_{2} \operatorname{gr} f_{1}-6 f_{1}^{2} \operatorname{gr} f_{2}+\right.$ $\left.8 f_{3} \operatorname{gr} f_{1}+8 f_{1} \operatorname{gr} f_{3}\right)$. Now we begin to calculate $\Delta f_{i}, i=1,2,3 . \Delta f_{1}=\operatorname{div}\left(\operatorname{gr} f_{1}\right)=6 n$, $\Delta f_{2}=\operatorname{div}\left(\operatorname{gr} f_{2}\right)=\sum_{1 \leq i \leq 3,1 \leq j \leq n} \varepsilon_{i j}\left\langle D_{z_{i j}} \operatorname{gr} f_{2}, X_{i j}\right\rangle$, where $\varepsilon_{i j}=\left\langle X_{i j}, X_{i j}\right\rangle=-1$, if $1 \leq i \leq 3,1 \leq$ $j \leq p$ and $\varepsilon_{i j}=+1$ if $1 \leq i \leq 3, p+1 \leq j \leq n$, if we write the points of $\bar{M}$ as

$$
x=\left[\begin{array}{l}
x_{11} \ldots x_{1 n} \\
x_{21} \ldots x_{2 n} \\
x_{31} \ldots x_{3 n}
\end{array}\right] .
$$


Then $X_{i j}: \bar{M} \rightarrow T \bar{M}, T_{z} \bar{M} \cong \bar{M} \cong \mathbb{R}_{3 p}^{3 n}$ is defined by $X_{i j}(x)=$ the $3 \times n$ matrix with its $(i, j)$ component one and other components zero. Thus $\left\{X_{i j}: 1 \leq i \leq 3,1 \leq j \leq n\right\}$ is an orthonormal frame field on $\bar{M}$. So we have

$$
\Delta f_{2}(x)=\sum \varepsilon_{i j}\left\langle X_{i j}(x) J x^{t} x+x J X_{j i}(x) x+x J x^{t} X_{i j}(x), X_{i j}(x)\right\rangle=(3 n+2) f_{1} .
$$

Similarly we obtain that $\Delta f_{3}=(3 n+4) f_{2}$. Thus $f$ is an isoparametric map. It is an interesting problem to study the geometry of nondegenerate regular levels of $f$ and also the focal manifolds associated with these regular levels.

The following is an example of an isoparametric submanifold of codimension 2 in $\mathbb{R}_{n+1}^{2 n+2}$ which has complex principal curvatures. It is sharply in contrast to the Riemannian case.

EXAMPLE 15. Let $f: \mathbb{R}_{n+1}^{2 n+2} \rightarrow \mathbb{R}^{2}$ be defined by

$$
f(x)=\left[\sum_{i=1}^{n+1} x_{1} x_{n+1+i}, \frac{1}{2}\left(-\sum_{i=1}^{n+1} x_{i}^{2}+\sum_{i=n+2}^{2 n+2} x_{i}^{2}\right)\right],
$$

let us check that $f$ is a (quadratic) isoparametric map and find a nondegenerate regular level of it.

Let

$$
A_{1}=\frac{1}{2}\left[\begin{array}{c|c}
0 & -I_{n+1} \\
\hline I_{n+1} & 0
\end{array}\right] \text { and } A_{2}=\frac{1}{2} I_{2 n+2}
$$

then $\left\{A_{1}, A_{2}\right\}$ is a linearly independent set in $\operatorname{Sym}\left[\mathbb{R}_{n+1}^{2 n+2}\right]$ and we have $A_{n+1}^{2}=-\frac{1}{4} I_{2 n+2}$ so $\left\{A_{1}, A_{2}\right\}$ generate a 2-dimensional algebra, it is easily seen that $f$ is a (quadratic) isoparametric map [3]. Consider the level $f^{-1}\left(\left(0, \frac{1}{2} b^{2}\right)\right), b \neq 0$. Since $b \neq 0$, obviously $\left\{A_{1} x, A_{2} x\right\}$ is a linearly independent set, so $f^{-1}\left(\left(0, \frac{1}{2} b^{2}\right)\right)$ is a regular level.

For the nondegeneracy of $f^{-1}\left(\left(0, \frac{1}{2} b^{2}\right)\right)$ we examine the system of equations

$$
\left\{\begin{array}{l}
\left\langle\alpha A_{1} x+\beta A_{2} x, A_{1} x\right\rangle=0=\alpha\langle x, x\rangle=\alpha b^{2} \Rightarrow \alpha=0, \\
\left\langle\alpha A_{1} x+\beta A_{2} x, A_{2} x\right\rangle=0=\beta\langle x, x\rangle=\beta b^{2} \Rightarrow \beta=0 .
\end{array}\right.
$$

So $f^{-1}\left(\left(0, \frac{1}{2} b^{2}\right)\right)$ is nondegenerate. If $M$ is a component of $f^{-1}\left(\left(0, \frac{1}{2} b^{2}\right)\right)$ then $M$ is a quadratic isoparametric submanifold of codimension 2 and signature $(n, n)$. The shape operator of $M$ along $\left(-A_{2} x\right)$ is $\left.A_{2}\right|_{r M}$, which is real and diagonal and the shape operator of $M$ along $-A_{1} x$ is $\left.A_{1}\right|_{T M}$. Note that $A_{1}^{2}=-\frac{1}{4} I_{2 n+2}$ hence the minimal polynomial of $A_{1}$ is $t^{2}+\frac{1}{4}$. So $A_{1}$ has no real eigenvalue, but complex eigenvalues $\pm \frac{1}{2} i$, thus $\left.A_{1}\right|_{T M}$ has no real eigenvalue.

ACKNowledgement. The author wishes to acknowledge the hospitality of the Sharif University of Technology for the typing of the paper.

\section{REFERENCES}

1. S. Carter and A. West, Isoparametric systems and transnormality. Proc. London Math. Soc. (3), 51 (1985), 520-542.

2. J. Hahn, Isoparametric hypersurfaces in the pseudo Riemannian space forms. Math. Z. 187 (1984), 195-208. 
3. S. M. B. Kashani, Isoparametric submanifolds in pseudo Riemannian spaces. Thesis, Leeds University, 1988.

4. K. Nomizu, Elie Cartan's work on isoparametric families of hypersurfaces, Proc. of Symposia in Pure Math. (AMS) 27 (1975), 191-200.

5. C. L. Terng, Isoparametric submanifolds and their Coxeter groups. J. Diff. Geom. 21 (1985), 79-107.

Mathematics Department

Sharif University of Technology

P.O. BOX 11365-9415

TEHRAN, Iran 